\title{
InNovative Course Scheduling And Curriculum Design
}

\author{
Ali Akgunduz, Yong Zeng \\ Faculty of Engineering and Computer Science \\ Concordia University, Montreal, Quebec \\ ali.akgunduz@concordia.ca; yong.zeng@concordia.ca
}

\begin{abstract}
Course scheduling is a challenging operations research problem that involves students, faculty members, availability of classrooms, class sizes and many other factors. As it is the case for most scheduling problems, course scheduling is an NP-Hard problem. Due to its challenging nature, frequently the main objective is only to find a feasible solution that satisfies students, faculty and classroom requirements rather than seeking the optimality which results in most effective teaching environment for most students. Such optimal learning environment requires the satisfaction of additional constraints such as the learning capacity of students and the capacity requirements of courses. In this research we investigate the possibility of quantifying course (curriculum) workload and the acquired capacity and suggest as curriculum and/or schedule design methodology that enables a near optimum learning environment for most students.
\end{abstract}

Keywords: Course Scheduling; Mental stress, Cognition capacity; Mathematical programming; Multi-criteria decision making.

\section{INTRODUCTION}

Cognition and education sciences suggest that, stress level and/or mental-workload significantly impact on the students' success. Students perceive knowledge more efficiently when their stress level is at the optimal level. While several factors impact on the stress and mental workload such as time of the day/week and various environmental factors (relationship with others, financial situations etc.), expected workload and student's learning capacity are the two main factors directly related to university learning experience. Cognition science defines stress as a ratio between expected workload and the mental capacity. Furthermore, the mental capacity consists of two parts: knowledge; and skills. Hence, a successful engineering curriculum design and course scheduling should satisfy not only the traditional constraints (students, classrooms and professors) but also aim at sustaining a stress level that foster highest possible student cognition.

The current CEAB criteria for accreditation have motivated all engineering programs to map their courses on graduate attributes. A student's performance in a class is the reflection of his/her comprehension level of the relevant graduate attributes. Comprehension level on the other hand depends on the stress level. Finally the stress is a ratio between course expectations and the student's capacity which is the knowledge and skills already acquired. In this research we investigate the possibility of quantifying course (curriculum) workload and the acquired capacity and suggest a curriculum and/or schedule design methodology that enables a near optimum learning conditions for most students.

\section{LITERATURE REVIEW}

Most general form of course scheduling problems deal with the assignment of courses to available classrooms according to faculty members' availabilities and students' course sequences. Usually some forms of penalty functions are incorporated to maximize student course selection choices [3]. Other objectives that may be of concern are faculty satisfaction, classroom utilization and class-size management. Regardless of the type objectives defined, the general methodology for tackling a course scheduling problem is addressed through variation of network optimization or assignment problems. In this paper on the other hand, we are interested in adding another dimension to the course scheduling problem: effective usage of student-attention-span (circadian rhythm and mental stress). Our main interest is to reduce the student stress level, align the courses according to students' available mental capacities and consequently increase the success level.

In literature, it is documented that circadian rhythm has strong correlation with the cognition capacity of individuals [8]. Randler and Frech studied university students aged 20-22 years to test if there is a correlation 
between biological-clock and academic performance [7]. Their results showed strong correlation between biological-clock and the academic performance. These results gave the inspiration to the work presented in this paper.

Randler and French grouped students in two categories as morningness to eveningness [7]. More recent works show that, cognition varies during a day significantly. Therefore, the cognition capacity of an individual at 10 AM may be significantly higher than at 3 PM. Such relationships have been established in the work of Blatter and Cojochen as illustrated in Fig. 1 [2].

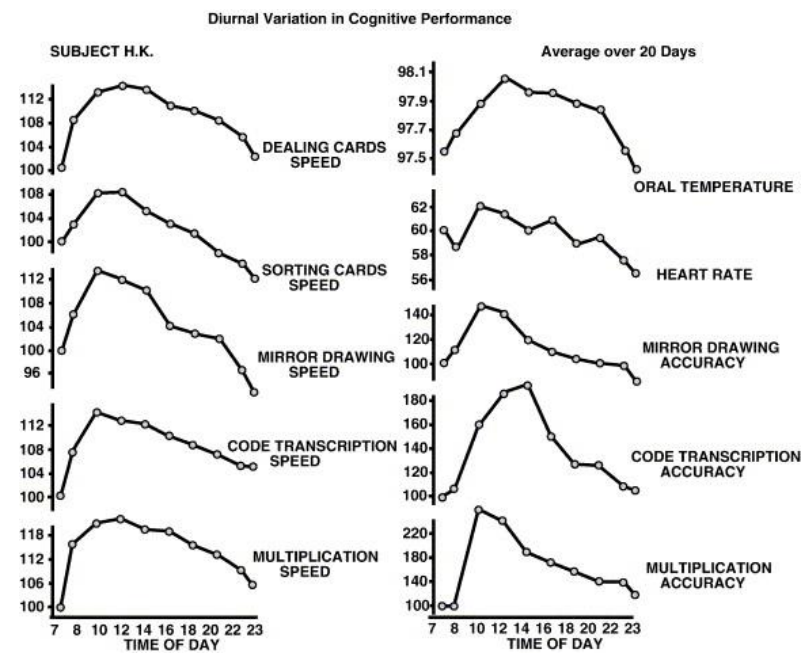

Fig. 1: Cognition capacity of individuals performing various tasks [2]

Combining both works -the notion of academic performance has strong correlation with the circadian rhythm (the work of Randler and French [7]) and the principle that circadian rhythm varies during the day (the work of Blatter and Cojochen [2]), enables us to make following claim: a course scheduling model that incorporates circadian rhythm would lead to a more effective learning environment. Furthermore, studies have suggest that, quantifying mental stress, workload and mental capacity foster students' creativity and enhance learning experience [4]. Hence, in this paper we modeled the course scheduling problem as a function of circadian rhythm and difficulty level of courses. The objective is to schedule the courses in such way that courses requires higher attention-span are scheduled at timeslots where the cognition capacity is higher and schedule the course that requires less attention-span at timeslot where cognition capacity is lower. Operations research provides a perfect environment to successfully incorporate these conditions in the course scheduling process.

\section{METHODOLOGY}

In order to successfully incorporate the stress factor, cognition capacity and course workload requirement in the course scheduling problem, following assumptions are made.

- Student success is influenced by the scheduled time of the course.

- Difficult courses are influenced more by the circadian rhythm

- Stress plays an important role in learning

- $\quad$ Stress can be defined as a function of Workload and Mental capacity

- Faculty members can objectively rank courses according to their difficulty levels

- The majority of student population follow a common circadian rhythm

While some of the aforementioned issues will only be treated as assumptions since we do not have sufficient data to verify the accuracy, we will attempt to prove the validity of others in the following sections.

\subsection{Student Success is Influenced by Course's Scheduled Time-of-the-day and Stress Level}

In their works, Randler and French have shown the correlation between the students' academic performances and their circadian rhythm [7]. Moreover, researchers like Blatter and Cojochen demonstrated that circadian rhythm changes during a day significantly [2]. Consequently we can claim that learning capacity of an individual changes during a day due to his/her circadian rhythm. If a course is scheduled during a time-slot where students' cognition capacities are higher (11 AM in the morning) but the success level of the class is lower, than we conclude that workload for the course is significantly higher than the current mental capacities of students. In other word, Knowledge and skill-sets students have at the moment is not matching the required workload. The mismatch between the workload and mental capacity is leading to higher mental stress. Consequently, student performance is lower.

In their works, Nguyen and Zeng, defined mental stress (MS) as a function of Workload (W) and Mental capacity (C) [5]. Mental capacity is further defined by its sub-members Knowledge (K) and Skill (S) and Environmental affects (A).

$M S=\frac{W}{C}=\frac{W}{(K+S) A}$

Nguyen and Zeng developed an experiment with seven subjects to investigate the link between mental effort and stress level [6]. Students were asked to tackle various challenging design problems. The results of their work show that individuals have less mental effort when the stress level is high. During this experiment, stress 
levels of students were measured using EEG signals. One of the factors that may be having an impact on the high stress levels is the lack of prior knowledge on the topic or lacking skill levels to tackle the course content. It is known that, when individuals tackle problems without sufficient background on the topic usually react to situation with higher stress levels.

Aforementioned examples enable us to make following conclusions:

- Circadian rhythm impacts cognition performance

- $\quad$ Prior knowledge on topic and skill level impacts on mental effort

- If the class performance (average GPA) is less than expectations, it can be explained by two reasons:

- Course is scheduled during the time-ofthe-day when the cognitive performance of individual is lower according to the circadian rhythms.

- Course sequence is misaligned and students do not have the sufficient knowledge to tackle the expected workload, therefore they have higher stress levels.

\subsection{Improved Course Scheduling}

In this study, we claim that scheduling courses according to the circadian rhythms will lead to a better learning environment. The challenge is, there are only a limited number of hours available in a given week and all courses have to be scheduled during acceptable time-of-the-days (typically from 8:45 AM till 6:45 PM for undergraduate courses at Concordia University). Therefore, schools have to schedule courses early in the morning and later in the afternoon when student cognition capacity is lower (see Fig. 2). According to the work of Nguyen and Zeng, it is known that, if the workload of students (in this case the difficulty level of a course) is lower, then the students' stress levels will be lower and consequently the mental effort will be higher [5]. Hence, scheduling a less difficult (less demanding) course during the time when individuals' cognition capacity is lower will not impact the student performance level. Based on this argument, we propose to model the course scheduling problem in such way that, courses with higher workload expectations to be scheduled for the timeslots when individuals have higher cognition capacity, and the courses with less workload requirement to be scheduled at timeslot when cognition capacity is lower. The resulting schedule will lead to a much productive learning environment.

\subsection{Improved Curriculum Design}

Once an optimum course scheduling is achieved, it is expected that student performance in courses are improved. However, in reality, a number of courses may still be the outliners and students continue performing poorly despite these courses are scheduled in the optimal timeslots. This can be explained by the misaligned curriculum where students are lacking from the necessary background content. Recall equation 1, if the student stress is high (because the ratio between required course workload and the necessary knowledge and skills is high), students' learning capacity is lower [7]. If we make the assumption that skill levels of all students in a class are compatible, therefore, the high stress level can be explained by the lack of background knowledge. In order to improve the learning environment even further, schools with such problems should revise their curriculum maps to identify the knowledge deficiencies through graduate attributes and make necessary curriculum changes and/or prerequisite changes.

\section{MATHEMATICAL MODEL FOR COURSE SCHEDULING}

In this section we incorporate the relationship between circadian rhythm and cognition capacity into a course scheduling problem. Let us assume that the relationship depicted on Fig. 2 is the general trend of circadian rhythm's impact on learning performance.

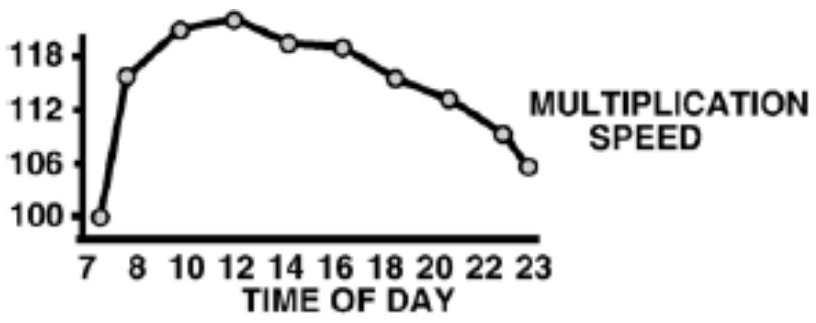

Fig. 2: Impact of circadian rhythm on learning performance [2]

In Fig. 3, a given school day is partitioned as timeslots to accommodate class-times (at Concordia University, 1 hour 15 minutes, twice a week policy is used for undergraduate courses)

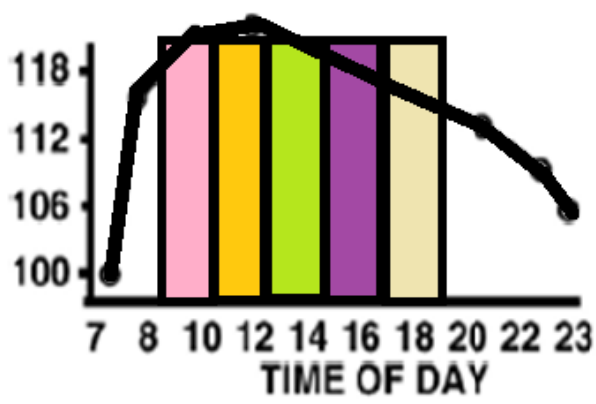

Fig 3. Available timeslots for undergraduate courses 
Based on the Fig. 3, there are 5 timeslots available for course scheduling for undergraduate students. Fig. 3 further suggests that more demanding courses should be scheduled in the 11:30-13:00 timeslots. Those courses with second degree of difficulty levels should be scheduled either at $10 \mathrm{AM}$ or $1 \mathrm{PM}$ time slots. Finally courses with lower difficulty levels can be scheduled in the remaining timeslots.

At this point one more remaining questions left to be addressed, the determination of course difficulty levels. In order to group the courses according to their difficulty levels, we propose a two-steps approach. First, rank courses according to their difficulty levels based on the professors and student feedback. Next, verify the rankings through course overall GPAs. In this study we use multi criteria decision making methodology, Analytic Hierarchy Process (AHP) to obtain professor and student feedback [1].

For the AHP, we sorted a number of courses according to averages class GPAs. We used two courses with low GPAs, 1 course with median and 2 courses with high GPAs in our example. We asked the faculty members and students to make a pairwise comparison between course pairs. Course instructors and students used following numerical values to quantify the qualitative values (Table 1).

Table 1: AHP Pairwise comparisons scales.

\begin{tabular}{|l|l|}
\hline Course 1 and Course 2 are equal in difficulty & 1 \\
\hline Course 1 is moderately more difficult than Course 2 & 3 \\
\hline Course 1 is strongly difficult than Course 2 & 5 \\
\hline Course 1 is very strongly difficult than Course 2 & 7 \\
\hline Course 1 is absolutely difficult than Course 2 & 9 \\
\hline
\end{tabular}

Table 2: Sample AHP ranking of a course instructor.

\begin{tabular}{|l|c|c|c|c|c|}
\hline & $\begin{array}{l}\text { ENGR } \\
\mathbf{2 1 4}\end{array}$ & $\begin{array}{l}\text { ENGR } \\
\mathbf{2 2 4}\end{array}$ & $\begin{array}{l}\text { ENGR } \\
\mathbf{2 5 0}\end{array}$ & $\begin{array}{l}\text { ENGR } \\
\mathbf{2 7 0}\end{array}$ & $\begin{array}{l}\text { ENGR } \\
\mathbf{2 9 0}\end{array}$ \\
\hline ENGR 214 & 1 & 3 & 5 & $1 / 5$ & 5 \\
\hline ENGR 224 & $1 / 3$ & 1 & 1 & $1 / 5$ & 5 \\
\hline ENGR 250 & $1 / 5$ & 1 & 1 & $1 / 9$ & 1 \\
\hline ENGR 270 & 5 & 5 & 9 & 1 & 9 \\
\hline ENGR 290 & $1 / 5$ & $1 / 9$ & 1 & $1 / 9$ & 1 \\
\hline
\end{tabular}

In Table 2, inverse ratios suggest that Course 2 is more difficult than Course 1. Using the mathematics suggested in AHP literature [1], courses are ranked according to their difficulty levels. An example is provided in Table 3.

Table 3: AHP Ranking

\begin{tabular}{|l|c|}
\hline Courses & AHP Ranking Weight \\
\hline ENGR 270 & 0.35 \\
\hline ENGR 214 & 0.22 \\
\hline
\end{tabular}

\begin{tabular}{|l|l|}
\hline ENGR 224 & 0.19 \\
\hline ENGR 250 & 0.13 \\
\hline ENGR 290 & 0.11 \\
\hline Total Weight & 1.00 \\
\hline
\end{tabular}

\subsection{Mathematical Model}

Let us assume that there are $\mathrm{N}$ courses to be scheduled in $\mathrm{M}$ classrooms and there are $\mathrm{L}$ timeslots available during a week. According to AHP method, courses are ranked based on their difficulty levels where $d_{n}$ is the quantified value for the difficulty. Furthermore, each available timeslot has quantified available mental capacity $\left(c_{l}\right)$ which is derived from Fig. 2 . The value of $c_{l}$ is normalized and range from 0 to $1\left(c_{l=\{11 A M\}}=1\right)$ and remaining timeslots are normalized accordingly using the scale in Fig. 2. Other decision variable is:

1 if course $n$ is scheduled in classroom $m$ $x_{n m l}=\quad$ in the timeslot $l$ 0 otherwise

\section{Objective Function}

$\min \sum_{n \in N} \sum_{m \in M} \sum_{l \in L} x_{n m l}\left(c_{l}-d_{n}\right)$

Model constraints

$\sum_{m \in M} \sum_{l \in L} x_{n m l}=2 \quad \forall n \in N$
$\sum_{n \in N} \sum_{l \in L} x_{n m l} \leq 1 \quad \forall m \in M$
$x_{n m l}=\sum_{m \in M} \sum_{l^{\prime} \in L(l)} x_{n m l^{\prime}} \quad \forall n \in N, l \in L$

where $L(l)$ refers to a timeslot that is same time as $l$ but in a different day of the week (each course is scheduled twice a week at the same time.

In the objective function, we only consider the minimization of difference between theoretical mental capacity and the difficulty level of a course. Equation (2) can be further enhanced to cover various exception cases. If the number of timeslots and available classrooms is tow high in comparison to number of courses to be scheduled, model may schedule the courses with lower difficulty levels on timeslots where the mental capacity is low even though there are still availabilities on high mental capacity timeslots. However, in practice such cases rarely to occur.

Equation 3 guarantees that each class is scheduled twice in a week. Equation 4 ensures that there is no double booking at a classroom. Finally in Equation 5, it is guaranteed that courses are scheduled at the same timeslots, twice in every week. 
As mentioned earlier, course scheduling can be modeled in variety of formats to accommodate various needs. In this study, we take a simple assignment modeling approach and put further emphasize on the discussion around the objective function.

\section{CONCLUSIONS}

In this paper, we introduce an innovative course scheduling and curriculum improvement procedure using operations research and cognitive science literature. In the paper, we successfully linked the relationship between mental stress and learning process. Moreover, we discuss the impact of the time-of-the-day on the learning process. Finally, we argued that if the courses are scheduled according to their difficulty levels and the available cognitive capacity of students, more effective learning environments can be achieved. We propose to achieve such course scheduling through operations research and AHP. Finally, using available literature we demonstrated that if the learning environment is not improved as a result of optimized scheduling, there must exists a misalignment in the program curriculum and it should be addressed through content and curriculum changes according to graduate attributes.

In the near future, we intend use the described mathematical model to determine an optimal course scheduling. Our preliminary data shows that, some of the most difficult courses are already scheduled in timeslots where we expect the students to have optimal learning conditions. Due to lack of grad distribution data, we were not able to report the impact of mental stress on grades as part of this study. In the near future we intend to report the impact of mental stress on academic performance at engineering schools as well.

\section{References}

[1] Akgunduz A, Zetu D, Banerjee P, and Liang D, "Evaluation of Sub-component Alternatives in Product Design Process", Robotics and Computer Integrated Manufacturing, 18 (1): 69-81, 2002 .

[2] Blatter K, Cajochen C, "Circadian Rhythms in Cognitive Performance: Methodological Constraints, Protocols, Theoretical Underpinnings", Physiology \& Behaviours 90:196-208, 2007.

[3] Dinkel JJ, Mote J and Venkataramanan MA, "An Efficient Decision Support System for Academic Course Scheduling, Operations Research, 37(6):853864, 1989.

[4] Jensen E, Brain-based learning: The new paradigm of teaching, California, Sage Publication, 2008.

[5] Nguyen TA, Zeng Y, "Effects of Stress and Effort on Self-rated Reports in Experimental Study of Design Activities", Journal of Intelligent Manufacturing, 2016.

[6] Nguyen TA, Zeng Y, "A Philosophical Study of Relationship Between Designer's Mental Effort and Mental Stress During Conceptual Design", Computer Aided Design, 54:3-18, 2014

[7] Randler C, Frech D, "Correlation Between Morningness-Eveningness and Final School Leaving Exams", Biological Rhythm Research, 35(3): 233 239, 2006

[8] Sousa D, "Impact of Circadian Rhythms on Schools and Classrooms", How the Brain Learns, Educational Neuroscience for Teachers, 2011. 